\title{
Factors influencing the characteristics dependence of zonal structure rock failure around lined opening
}

\author{
Lyudmila Ksendzenko ${ }^{1^{*}}$ \\ ${ }^{1}$ Far Eastern Federal University, Vladivostok, Russia
}

\begin{abstract}
For the safe mining operations, it is necessary to take into account a large number of negative factors, one of which is zonal failure (disintegration) of rocks that occurs after developing deposits at great depths.Therefore, investigation of the behavior of zonal mesostructures regularities around deep mine openings is relevant.In this paper dependences from influencing factors of the first failure zone radial extent, of the depth of the contour zone and the first failure zone merging, and the depth of the second failure zone appearance are established based on the non-Euclidean model of the zonal failure rock mass around deep lined openings and the Odintsev's criterion of the failure under compression. The influencing factors are the uniaxial compression strength of the rock $\sigma_{c}(\mathrm{MPa})$; the opening depth $\mathrm{H}(\mathrm{m})$; the ratio of empirical coefficients $\gamma_{3} / \gamma_{1}$; the values of the rocks elastic modulus, E, and the Poisson's ratio, $v$. The study was carried out both for hard and weak rocks at values resistance of support 0.5 and $1 \mathrm{MPa}$. Key words: lined opening, rock mass, zonal failure, non-Euclidean model, depth of zones merging
\end{abstract}

\section{Introduction}

The disruption of the massif solidity at great depths has a periodic, zonal character, in the literature it is known as "zonal disintegration." Under these conditions, the deformation of the surrounding rocks also acquires an oscillatory periodic character.The rock massif is a hierarchically-block medium $[1,2]$.

The description of the block defective media behavior within the framework of classical models of continuum mechanics encounters fundamental difficulties: 1) the inability to take into account the conditions for the disruption of the continuity of the medium as a result of large compressive stresses action; 2) the difficulty of representing the rock mass by a medium far from the conditions of thermodynamic equilibrium, and others. The influence lining of the opening on the character of the massif zonal destruction has not been adequately studied at present. To ensure the ability to manage the characteristics of the failure zonal structure, depending on the values of the influencing factors, special evaluation studies are needed.

\footnotetext{
${ }^{*}$ Corresponding author: ksendzenko@mail.ru
} 
In the paper, the dependences of some characteristics zonal structure of the rock massif failure around the lined opening from the influencing factors are investigated. A defective field around a lined underground opening of a circular section corresponding to the mesolevel of the massif [3] is described by a non-Euclidean model, where the stress components are determined by the relationships [4]:

$$
\begin{gathered}
\sigma_{r r}=\sigma_{\infty}\left(1-r_{0}^{2} / r^{2}\right)-\left(E / 2\left(1-v^{2}\right) \gamma^{1,5} r\right)\left\{a J_{1}(r \sqrt{\gamma})+b N_{1}(r \sqrt{\gamma})+c K_{1}(r \sqrt{\gamma})-\right. \\
\left.-\left(r_{0} / r\right)\left[a J_{1}\left(r_{0} \sqrt{\gamma}\right)+b N_{1}\left(r_{0} \sqrt{\gamma}\right)+c K_{1}\left(r_{0} \sqrt{\gamma}\right)\right]\right\}+P\left(r_{0}^{2} / r^{2}\right) \\
\sigma_{\varphi \varphi}=\sigma_{\infty}\left(1+r_{0}^{2} / r^{2}\right)-\left(E / 2\left(1-v^{2}\right) \gamma\right)\left(a J_{0}(r \sqrt{\gamma})+b N_{0}(r \sqrt{\gamma})-c K_{0}(r \sqrt{\gamma})\right)-P\left(r_{0}^{2} / r^{2}\right)+ \\
+\frac{E}{2\left(1-v^{2}\right) \gamma^{1,5} r}\left\{a J_{1}(r \sqrt{\gamma})+b N_{1}(r \sqrt{\gamma})+c K_{1}(r \sqrt{\gamma})-\frac{r_{0}}{r}\left[a J_{1}\left(r_{0} \sqrt{\gamma}\right)+b N_{1}\left(r_{0} \sqrt{\gamma}\right)+c K_{1}\left(r_{0} \sqrt{\gamma}\right)\right]\right\} .
\end{gathered}
$$

Here $\mathrm{P}$ is the resistance of support $(\mathrm{MPa}) ; \sigma_{\infty}=\gamma_{\mathrm{r}} \mathrm{\Psi H}, \gamma_{\mathrm{r}}$ is the unit weight of rock $\left(\mathrm{kN} / \mathrm{m}^{3}\right)$, and $\mathrm{H}$ is the opening depth $(\mathrm{m}) ; \boldsymbol{r}_{\boldsymbol{0}}$ is the radius of opening $(\mathrm{m}) ; \boldsymbol{r}$ is the distance from the center of opening to the current point of the rock mass (m); $\boldsymbol{E}$ is the modulus of elasticity $(\mathrm{MPa}) ; v$ is the Poisson's ratio; $\gamma, \mathrm{c}$ are the model parameters $\left(\mathrm{m}^{-2}\right) ; \mathrm{J}_{0}, \mathrm{~N}_{0}, \mathrm{~K}_{0}, \mathrm{~J}_{1}$, $\mathrm{N}_{1}, \mathrm{~K}_{1}$ are the Bessel, Neumann, Macdonald functions of zero and first orders, respectively.

At the same time, cracks related to the macro level for the rock massif are considered not to be interacting, and the failure conditions defined in [5] are applicable to them:

$$
K_{1}=\sqrt{\pi l}\left(\gamma_{1} \sigma_{\varphi \varphi}-\gamma_{3} \sigma_{r r}\right) \geq K_{1 c},
$$

where $l$ is the half-length of the rock mass fracture faults and is assumed to be equal to the minimum half-length of a tensile macrocrack (m), which his unstable in stress conditions; $\sigma_{\varphi \varphi}, \sigma_{r r}$ are the maximum and minimum principal stresses, respectively (MPa)determined in (1), (2); $\gamma_{1}$ and $\gamma_{3}$ are the empirical factors; $\mathrm{K}_{1}$ is the coefficient of stress intensity $\left(\mathrm{M \Pi a} \cdot \mathrm{M}^{1 / 2}\right) ; \mathrm{K}_{\mathrm{lc}}$ is the fracture toughness of rock material $\left(\mathrm{M \Pi a} \cdot \mathrm{M}^{1 / 2}\right)$. The resistance of the support $\mathrm{P}$ is interpreted as the magnitude of the radial stresses at the contact of the support with the rock mass.

\section{Hard rocks zonal failure around a lined opening regularities investigation}

The following characteristics of the zonal structure of the rock massif failure around deep rock openings are investigated: the radial extent of the first failure zone, the depth of the contour zone and the first failure zone merging, and the depth of the second fracture zone appearance at the values resistance of the support $\mathrm{P}=0.5$ and $\mathrm{P}=1 \mathrm{MPa}$ of rocks at Nikolaevskij mine (Dalnegorsk, Primorsky Krai, Russia) (Table 1).

Table 1. Physical and mechanical characteristics of the studied rocks

\begin{tabular}{|c|c|c|c|c|c|}
\hline Type of rocks & $\begin{array}{c}\text { UCS } \\
(\mathrm{MPa})\end{array}$ & $\begin{array}{c}\text { Young's } \\
\text { modulus } \\
(\mathrm{MPa})\end{array}$ & $\begin{array}{c}\text { Poisson } \\
\text { ratio }\end{array}$ & $\begin{array}{c}\text { Fracture } \\
\text { toughness } \\
\left(\mathrm{MPa}_{\mathrm{m}} \mathrm{m}^{0.5}\right)\end{array}$ & $\begin{array}{c}\text { Volume } \\
\text { weight } \\
\left(\mathrm{kN} \cdot \mathrm{m}^{-3}\right)\end{array}$ \\
\hline The hard rock & $100-250$ & $17000-35000$ & $0.15-0.26$ & $1.25-2.5$ & $26-28$ \\
\hline
\end{tabular}




\subsection{The first failure zone radial extent dependence on the influencing factors for hard rocks}

Dependence of the radial extent of the first fracture zone on the depth of the tunneling for different values of uniaxial compressive strength $\sigma_{\mathrm{c}}$ of the rock and the ratio of the empirical coefficients $\gamma_{3} / \gamma_{1}$ for the resistance of the supports $\mathbf{P}=0.5$ and $\mathbf{P}=1 \mathrm{MPa}$ is reflected in Tables 2, 3, 4 .

It is established that with an increase of opening depth, the radial extent of the first failure zone increases and the faster, the less the ratio $\gamma_{3} / \gamma_{1}$ (Table 2, 3, 4). The radial extent of the first failure zone increases with increasing opening depth, the faster, the less the strength of the rock on uniaxial compression was established. The radial extent of the first fracture zone is shown to decrease if resistance of the support rises, but insignificantly (Tables 2, 3, 4). For example, at $\sigma_{c}=100 \mathrm{MPa}$ (Table 2), the divergence in the radial extent of the first failure zone does not exceed $4 \%$ at values resistance of support $\mathrm{P}=0.5$ and $\mathrm{P}=1 \mathrm{MPa}$.

The influence of the support resistance decreases if the strength of the rock on uniaxial compression rises (Tables 2, 3, 4). It is shown if the ratio $\gamma_{3} / \gamma_{1}$ rises, the radial extent of the first failure zone decreases the faster, the less the strength of the rock on uniaxial compression at a fixed depth. The radial extent of the first failure zone is established to decrease if ratio $\gamma_{3} / \gamma_{1}$ increases and the faster it is, the greater the opening depth (Tables 2, 3, 4).

Table 2. Comparison of the radial extent of the first failure zone for resistance of supports 0.5 and 1 $\mathrm{MPa}$, different opening depths, ratios $\gamma_{3} / \gamma_{1}$ and fixed values $\sigma_{\mathrm{c}}=100 \mathrm{MPa}$

\begin{tabular}{|c|c|c|c|c|}
\hline \multirow{2}{*}{$\mathrm{P}, \mathrm{MPa}$} & \multirow{2}{*}{$\mathrm{H}, \mathrm{m}$} & \multicolumn{3}{|c|}{$\gamma_{3} / \gamma_{1}$} \\
\cline { 3 - 5 } & & 0.7 & 0.8 & 0.9 \\
\hline 0.5 & 450 & 0.349732 & 0.293720 & 0.247328 \\
\hline 1 & 450 & 0.342596 & 0.288524 & 0.243385 \\
\hline \multicolumn{2}{|c|}{ The divergence, \% } & -2.04 & -1.8 & -1.6 \\
\hline 0.5 & 550 & 0.639650 & 0.527899 & 0.440048 \\
\hline 1 & 550 & 0.623972 & 0.517409 & 0.432437 \\
\hline \multicolumn{2}{|c|}{ The divergence, \% } & -2.5 & -2.0 & -1.7 \\
\hline 0.5 & 650 & 0.906963 & 0.715899 & 0.586888 \\
\hline \multicolumn{2}{|c|}{ The divergence, $\%$} & 0.871841 & 0.698732 & 0.575536 \\
\hline \multicolumn{2}{|l|}{$\%$} & -3.9 & -2.4 & -1.9 \\
\hline
\end{tabular}

Table 3. Comparison of the radial extent of the first failure zone for resistance of supports 0.5 and $1 \mathrm{MPa}$, different opening depths, ratios $\gamma_{3} / \gamma_{1}$ and fixed values $\sigma_{\mathrm{c}}=120 \mathrm{MPa}$

\begin{tabular}{|c|c|c|c|c|}
\hline \multirow{2}{*}{$\mathrm{P}, \mathrm{MPa}$} & \multirow{2}{*}{$\mathrm{H}, \mathrm{m}$} & \multicolumn{3}{|c|}{$\gamma_{3} / \gamma_{1}$} \\
\cline { 3 - 5 } & & 0.7 & 0.8 & 0.9 \\
\hline 0.5 & 600 & 0.393720 & 0.327695 & 0.271992 \\
\hline 1 & 600 & 0.388829 & 0.324128 & 0.269312 \\
\hline \multicolumn{2}{|c|}{ The divergence, $\%$} & -1.2 & -1.1 & -1.0 \\
\hline 0.5 & 800 & 0.680011 & 0.555470 & 0.455874 \\
\hline 1 & 800 & 0.670064 & 0.548771 & 0.451058 \\
\hline \multicolumn{2}{|c|}{ The divergence, \% } & -1.5 & -1.2 & -1.1 \\
\hline 0.5 & 1000 & 0.946749 & 0.742747 & 0.599215 \\
\hline \multicolumn{2}{|c|}{ The divergence, $\%$} & 0.926651 & 0.732255 & 0.592274 \\
\hline \multicolumn{2}{|c|}{$\%$} & -2.1 & -1.4 & -1.2 \\
\hline
\end{tabular}


Table 4. Comparison of the radial extent of the first failure zone for resistance of supports 0.5 and 1 $\mathrm{MPa}$, different opening depths, ratios $\gamma_{3} / \gamma_{1}$ and fixed values $\sigma_{\mathrm{c}}=180 \mathrm{MPa}$

\begin{tabular}{|c|c|c|c|c|}
\hline \multirow{2}{*}{$\mathrm{P}, \mathrm{MPa}$} & \multirow{2}{*}{$\mathrm{H}, \mathrm{m}$} & \multicolumn{3}{|c|}{$\gamma_{3} / \gamma_{1}$} \\
\hline & & 0.7 & 0.8 & 0.9 \\
\hline 0.5 & 800 & 0.300844 & 0.249089 & 0.202957 \\
\hline 1 & 800 & 0.299306 & 0.247940 & 0.202091 \\
\hline \multicolumn{2}{|c|}{ The divergence, $\%$} & -0.5 & -0.5 & -0.4 \\
\hline 0.5 & 1000 & 0.576396 & 0.473812 & 0.384159 \\
\hline 1 & 1000 & 0.573267 & 0.471533 & 0.382470 \\
\hline \multicolumn{2}{|c|}{ The divergence, $\%$} & -0.5 & -0.5 & -0.4 \\
\hline 0.5 & 1500 & 1.039442 & 0.830789 & 0.662640 \\
\hline 1 & 1500 & 1.032250 & 0.826183 & 0.659448 \\
\hline \multicolumn{2}{|c|}{ The divergence, $\%$} & -0.7 & -0.6 & -0.5 \\
\hline 0.5 & 1900 & 1.442766 & 1.077890 & 0.841364 \\
\hline 1 & 1900 & 1.422335 & 1.070620 & 0.836876 \\
\hline \multicolumn{2}{|c|}{ The divergence, $\%$} & -1.4 & -0.7 & -0.5 \\
\hline
\end{tabular}

\subsection{Dependence of the contour merging depth and the first failure zone on the influencing factors for hard rocks}

As the opening depth is increasing, the radial extent of the contour zone rises, and the radial extent of the first intermediate zone decreases to zero, which results in the merging of the contour zone and first zone of failure. Fig. $1 a, b$ shows the dependence of the depth merging of the first failure zone with the contour zone on the uniaxial compression strength at values of support resistance $\mathrm{P}=0.5$ and $\mathrm{P}=1 \mathrm{MPa}$, respectively.

It is established that with increasing in the rock uniaxial compression strength, the depth of merging of the contour and first failure zones increases and the faster it is, the larger the ratio $\gamma_{3} / \gamma_{1}$. The influence of the values of support repulse on the depth of merging of the contour and the first failure zones for different values $\sigma_{\mathrm{c}}$ and ratios $\gamma_{3} / \gamma_{1}$ is shown in Table. 5. It is shown that with a decrease in the uniaxial compression strength $\sigma_{c}$, the influence of the support on the depth of merging of the contour and the first failure zones becomes more significant, but the difference in the depth of merging does not exceed $4 \%$.

Dependences of the depth of merging of the contour and first failure zones on the ratio $\gamma_{3} / \gamma_{1}$ for different values of the rock uniaxial compression strength are shown in Fig. $1 c, d$. It is established that with increasing ratio $\gamma_{3} / \gamma_{1}$, the depth of merging of the contour and the first failure zones increases and the faster it is, the larger the value $\sigma_{c}$. At the same time, the influence of support resistance is insignificant (Table 5).

Table 5. The depth of merging contour and first failure zones $(\mathrm{m})$ at values the resistance of support $P$

$=0.5$ и $1 \mathrm{MPa}$, different values of the uniaxial compression strength of the rock and ratio $\gamma_{3} / \gamma_{1}$

\begin{tabular}{|c|c|c|c|c|c|c|}
\hline \multirow{3}{*}{$\gamma_{3} / \gamma_{1}$} & \multicolumn{6}{|c|}{$\sigma_{\mathrm{c}}, \mathrm{MPa}$} \\
\hline & \multicolumn{2}{|c|}{100} & \multicolumn{2}{|c|}{120} & \multicolumn{2}{|c|}{180} \\
\hline & $\mathrm{P}=0.5 \mathrm{MPa}$ & $\mathrm{P}=1 \mathrm{MPa}$ & $\mathrm{P}=0.5 \mathrm{MPa}$ & $\mathrm{P}=1 \mathrm{MPa}$ & $\mathrm{P}=0.5 \mathrm{MPa}$ & $\mathrm{P}=1 \mathrm{MPa}$ \\
\hline 0.7 & 814.4 & 841.6 & 1066.2 & 1087.5 & 1967.6 & 1986.6 \\
\hline 0.8 & 969.6 & 995.4 & 1274.1 & 1299.2 & 2416.6 & 2439.4 \\
\hline 0.9 & 1154.0 & 1184.2 & 1534.6 & 1564.2 & 3013.7 & 3041.6 \\
\hline
\end{tabular}



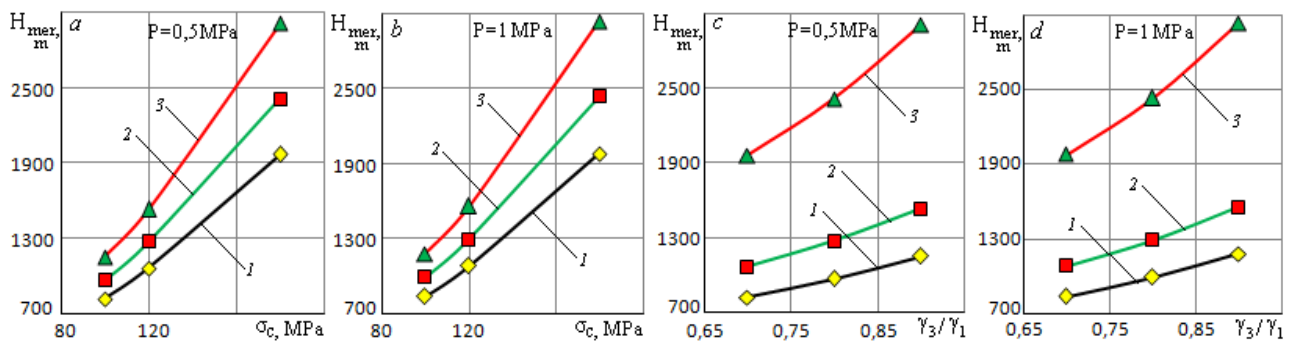

Fig. 1. Dependence of contour merging depth and first failure zones for hard rocks on: $a$ ) the uniaxial compression strength of the rock $\sigma_{c}$ at the values $\gamma_{3} / \gamma_{1}$, respectively: $1-0.7 ; 2-0.8 ; 3-0.9(a, b)$; the ratio $\gamma_{3} / \gamma_{1}$ at the values uniaxial compression strength of the rock $\sigma_{c}(\mathrm{MPa}): 1-120 ; 2-150 ; 3$ $-180(c, d) ;(a, c)$ - at resistance of support $\mathrm{P}=0.5 ;(b, d)-\mathrm{P}=1 \mathrm{MPa}$

\subsection{Dependence of the second failure zone depth appearance on the influencing factors for hard rocks}

Dependences of the depth at which the second zone of failure appears on the uniaxial compression strength of the rock at resistance of the supports $\mathrm{P}=0.5$ and $\mathrm{P}=1 \mathrm{MPa}$ respectively are shown in Fig. $2 a, b$. It is established that with increasing in the rock uniaxial compression strength, the depth at which the second zone of failure appears also increases. With an increase in the ratio $\gamma_{3} / \gamma_{1}$, the second zone of failure appears at a greater depth (Fig. $2 c, d$ ).

The influence of the values of support resistance on the depth at which the second zone of failure appears for different values $\sigma_{c}$ and ratios $\gamma_{3} / \gamma_{1}$ is shown in Table 6. It can be noted that with the decrease in the uniaxial compression strength of the rock $\sigma_{c}$, the effect resistance of support increases and that the difference in the depth at which the second zone of failure appears for resistance of supports $\mathrm{P}=0.5$ and $\mathrm{P}=1 \mathrm{MPa}$ does not exceed $1.5 \%$.
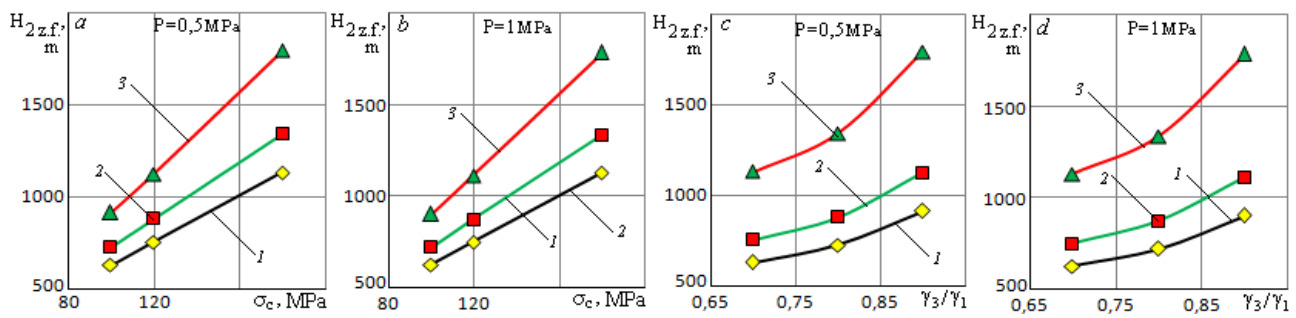

Fig.2. Depth dependence at which the second zone of failure appearsfor hard rocks on: $a$ ) the uniaxial compression strength of the rock $\sigma_{c}$ at the values $\gamma_{3} / \gamma_{1}$, respectively: $1-0.7 ; 2-0.8 ; 3-$

$0.9(a, b)$; the ratio $\gamma_{3} / \gamma_{1}$ at the values uniaxial compression strength of the rock $\sigma_{c}(\mathrm{MPa}): 1-120$; $2-150 ; 3-180(c, d) ;(a, c)-$ at resistance of support $\mathrm{P}=0.5 ;(b, d)-\mathrm{P}=1 \mathrm{MPa}$ 
Table 6. The depth at which the second zone of failure appears (m)

\begin{tabular}{|c|c|c|c|c|c|c|}
\hline \multirow{3}{*}{$\gamma_{3} / \gamma_{1}$} & \multicolumn{6}{|c|}{$\sigma_{\mathrm{c}}, \mathrm{MPa}$} \\
\hline & \multicolumn{2}{|c|}{100} & \multicolumn{2}{|c|}{120} & \multicolumn{2}{|c|}{180} \\
\hline & $\mathrm{P}=0.5 \mathrm{MPa}$ & $\mathrm{P}=1 \mathrm{MPa}$ & $\mathrm{P}=0.5 \mathrm{MPa}$ & $\mathrm{P}=1 \mathrm{MPa}$ & $\mathrm{P}=0.5 \mathrm{MPa}$ & $\mathrm{P}=1 \mathrm{MPa}$ \\
\hline 0.7 & 631.6 & 625.3 & 758.4 & 752.6 & 1134.8 & 1130.1 \\
\hline 0.8 & 730.9 & 722.2 & 884.2 & 876.0 & 1345.1 & 1338.4 \\
\hline 0.9 & 916.2 & 903.0 & 1128.7 & 1115.9 & 1796.4 & 1785.1 \\
\hline
\end{tabular}

\section{Weak rocks around a lined opening zonal failure regularities Investigation}

The study was carried out at coal mine named Artyom (Shkotovo settlement, Primorsky Krai, Russia) (Table 7).

Table 7. Physical and mechanical characteristics of the studied rocks

\begin{tabular}{|c|c|c|c|c|}
\hline Type of rocks & $\begin{array}{c}\text { UCS } \\
(\mathrm{MPa})\end{array}$ & $\begin{array}{c}\text { Young's modulus } \\
(\mathrm{MPa})\end{array}$ & $\begin{array}{c}\text { Poisson } \\
\text { ratio }\end{array}$ & $\begin{array}{c}\text { Fracture toughness } \\
\left(\mathrm{MPa} \times{ }_{\mathrm{m}}{ }^{\circ}\right)\end{array}$ \\
\hline The little hard rock & $8-15$ & $500-700$ & $0.14-0.16$ & $0.112-0.168$ \\
\hline
\end{tabular}

Fig. 3 shows the dependence the radial extent of the first fracture zone on the mining depth at the resistance of supports $\mathrm{P}=0.5$ and $\mathrm{P}=1 \mathrm{MPa}$, respectively. It is established that with an increase of mining depth, the radial extent of the first failure zone increases and the faster it is, the less the ratio $\gamma_{3} / \gamma_{1}$ (Fig. $3 a, b$ ). It can be seen from Figures $3 c, d$ that the radial extent of the first failure zone increases with increasing depth, and the faster it is, the less the $\sigma_{c}$.

It is shown that with increasing the resistance of the support, the radial extent of the first failure zone decreases and at a depth of $90 \mathrm{~m}$ the difference in the radial extent reaches $30 \%$ (Table 8). Hence, there is a significant influence the resistance of support on the radial extent of the first fracture zone for weak rocks.
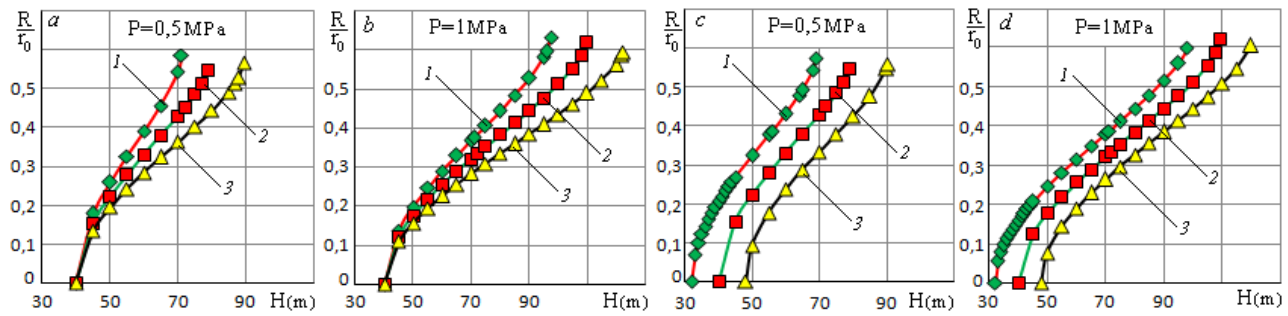

Fig. 3. Dependency graphs of the radial extent of the first failure zone on the opening depth for the weak rocks: $(a, b)-$ at ratio $\gamma_{3} / \gamma_{1}: 1-0.7 ; 2-0.8 ; 3-0.9, \sigma_{\mathrm{c}}=10 \mathrm{MPa}$ and values resistance of support $a-0.5 ; b-1 \mathrm{MPa} ;(c, d)$ - at ratio $\gamma_{3} / \gamma_{1}=0.8 ; \sigma_{\mathrm{c}}: 1-8 ; 2-10 ; 3-12 \mathrm{MPa}$ and values resistance of support $c-\mathrm{P}=0.5 ; d-\mathrm{P}=1 \mathrm{MPa}$

The patterns behavior of the rock massif zonal failure characteristics, established for hard rocks, is also preserved for the weak rocks. The influence of resistance of the support for the weak rocks is significant and reaches $30 \%$. It is found out that the deformation modulus $\mathrm{E}$ and the Poisson ratio have practically no effect on the radial extent of the first failure zone, both for hard and weak rocks. 
Table1 8. Dependence of the first failure zone radial extent on opening depth at resistance of the support $\mathrm{P}=0.5$ and $1 \mathrm{MPa}, \gamma_{3} / \gamma_{1}=0.8$ и $\sigma_{c}=12 \mathrm{MPa}$

\begin{tabular}{|c|c|c|c|}
\hline $\mathrm{H}(\mathrm{m})$ & $\begin{array}{c}\text { The resistance of the support } \\
\mathrm{P}=0.5 \mathrm{MPa}\end{array}$ & $\begin{array}{c}\text { The resistance of the support } \\
\mathrm{P}=1 \mathrm{MPa}\end{array}$ & $\begin{array}{c}\text { The } \\
\text { divergence, \% }\end{array}$ \\
\hline 50 & 0.0938 & 0.0766 & -18.4 \\
\hline 70 & 0.3349 & 0.2647 & -21.0 \\
\hline 90 & 0.5485 & 0.3859 & -29.6 \\
\hline
\end{tabular}

\section{The discussion of the results}

The notions of contour (near the opening boundary) intermediate zones and fracture zones are related to the coefficient of normal stress intensity $K_{1}$, which depends on the gravitational stress.

For a certain value of the gravitational stress, an inequality (3) is satisfied, which indicates the zones appearance of fracture. The maxima of the oscillating function $K_{1}$ correspond to the zones of failure of the rock massif, and the minima to the intermediate zones it is assumed. The contour zone of failure is meant the zone enclosed between the contour of the opening and the first intermediate zone.

In the future, it is necessary to investigate the dependence on the influencing factors of the zones number, the position of the last failure zone, and others.

\section{Conclusions}

1. The radial extent of the first zone of failure increases if the depth rises, up to the confluence of neighboring zones; the radial extent of the first fracture zone decreases if the $\sigma_{\mathrm{c}}$ rises; the radial extent of the first fracture zone decreases if the ratio $\gamma_{3} / \gamma_{1}$ rises.

2. The merging of the contour zone and the first fracture zone occurs at a greater depth if the $\sigma_{\mathrm{c}}$ rises; the depth of the merging of the zones increases if the ratio $\gamma_{3} / \gamma_{1}$ rises.

3. With increasing in the $\sigma_{c}$, the depth of appearance of the second fracture zone also rises. If the ratio $\gamma_{3} / \gamma_{1}$ rises, the second zone of failure appears at a greater depth.

4. The radial extent of the first fracture zone decreases if the resistance of support rises. The insignificant influence of the resistance of support on the characteristics of the zonal fracture structure for hard rocks and a noticeable influence for weak rocks is shown.

The author thank Professor Vladimir Makarov for his important recommendations in the process of writing this paper. The research is supported by a grant from the Ministry of Science and Higher Education of the Russian Federation No. RFMEFI58418X0034.

\section{References}

1. M.A. Sadovsky, DAN USSR, 247, 829. (1979)

2. P.V. Makarov, Physical Mesomechanics, 7, 25. (2004)

3. V.V. Makarov, M. A. Guzev, V.N. Odintsev, L.S. Ksendzenko, Journal of Rock Mechanics and Geotechnical Engineering, 8, 164. (2016) .doi.org/10.1016/j.jrmge.2015.09.010

4. L.S. Ksendzenko, GIAB, 8,364. (2007)

5. V.N. Odintsev, Rupture destruction of a brittle rocks mass (Moscow: IPKON, RAS, 1996) 